\title{
Effect of Hydrophobicity of Anionic Copolypeptides on the Structure and Functions of Cationic Bilayer Membrane
}

\author{
Masahiro HiguchI, Takatoshi Kinoshita, ${ }^{*}$ Akira TAKIZAWA, \\ and Yoshiharu TSUJITA \\ Department of Materials Science and Engineering, Nagoya Institute of Technology, \\ Gokiso-cho, Showa-ku, Nagoya 466, Japan
}

(Received September 27, 1988)

\begin{abstract}
The interactions between anionic random copolypeptides and cationic bilayer membrane composed of distearyldimethylammonium chloride (DSACl) were investigated and the effect of hydrophobicity of the anionic copolypeptide on these interactions was thermodynamically elucidated. The anionic copolypeptides, poly(L-glutamic acid)s containing various amounts of $\gamma$ methyl L-glutamate as a hydrophobic residue $(\mathrm{MG} / \mathrm{GA}(X / 100-X)), X$ is a mol\% of $\gamma$-methyl Lglutamate residues have been prepared by saponification reaction of poly $(\gamma$-methyl L-glutamate) (PMG, $\bar{M}_{v}=4400$ ) whose length along the $\alpha_{\text {-helix }}$ rod is almost comparable with the thickness ( $37 \AA$ ) of the bilayer membrane formed by DSACl. The partition of MG/GA into the DSACl membrane interior was shown to decrease by decreasing the degree of hydrophobicity of $\mathrm{MG} / \mathrm{GA}$. As a result, the incorporation of the copolypeptide could not occur with MG/GAs containing more than $30 \mathrm{~mol} \% \mathrm{~L}$-glutamic acid residue, which was almost localized at the membrane surface. It was shown that the concentration of $\mathrm{MG} / \mathrm{GA}$ in the bilayer membrane above $T_{\mathrm{c}}$ was higher than that below $T_{\mathrm{c}}$. Temperature dependence of the partition equilibrium of $\mathrm{MG} / \mathrm{GA}$ between the membrane surface and membrane interior also showed positive changes in enthalpy and entropy, respectively, accompanied by the incorporation of the copolypeptide into the membrane. It was also shown that the sodium ion permeability through the bilayer membrane had a maximum value when MG/GA with $12 \mathrm{~mol} \%$ L-glutamic acid residue was incorporated into the membrane.

KEY WORDS Low Molecular Weight Polypeptide / Poly $(\gamma$-methyl Lglutamate) / Poly( $\gamma$-methyl L-glutamate-co-L-glutamic acid) / Poly(Lglutamic acid) / Artificial Membrane Vesicle / Distearyldimethylammoniumchloride / Partition / Hydrophobic / Enthalpy and Entropy Change / Ionic Permeability /
\end{abstract}

The functions of a biological membrane, such as selective ion permeability ${ }^{1}$ and transmission of information between cells, ${ }^{2}$ are closely related to the interactions between membrane protein and the lipid bilayer membrane. Thus, studies of model systems composed of polypeptide and artificial bilayer membrane may be important to understand basic aspects of interactions between membrane protein and lipid bilayer membrane.

In a previous study, ${ }^{3}$ we investigated the partition of relatively low molecular weight polypeptides between bilayer membrane surface and the interior of the DSACl bilayer membrane by means of fluorescence spectroscopic technics. A hydrophobic polypeptide, poly ( $\gamma$-methyl L-glutamate) (PMG) was readily incorporated into the bilayer membrane interior and formed membrane-spaning helices. On the other hand, a negatively charged polypeptide, poly(L-glutamic acid) (PGA), could hardly penetrate the bilayer membrane and

\footnotetext{
* To whom correspondence should be addressed.
} 
formed crosslinking bridges between the positively charged DSACl vesicles. In addition, we have also shown ${ }^{4}$ that a photoresponsive polypeptide, azobenzene-modified poly $(\gamma$-methyl L-glutamate-co-L-glutamic acid) was transferred from the hydrophobic bilayer membrane interior to the hydrophilic surface by UV light $(<330 \mathrm{~nm})$ irradiation owing to the photoinduced polarity increase of the azobenzene moieties of the photoresponsive polypeptide.

We report here the effect of the degree of hydrophobicity of poly $(\gamma$-methyl L-glutamateco-L-glutamic acid)s (MG/GAs) on the partition of $\mathrm{MG} / \mathrm{GA}$ between the bilayer membrane surface and membrane interior and also on the ionic permeability through the bilayer membrane containing $\mathrm{MG} / \mathrm{GA}$ by using $\mathrm{MG} /$ GAs with various amounts of the L-glutamic acid residues.

\section{EXPERIMENTAL}

\section{Materials}

Polypeptides. Poly $(\gamma$-methyl L-glutamate) (PMG) was obtained by the polymerization of $N$-carboxy anhydride of L-glutamic acid $\gamma$ methyl ester in 1,2-dichloroethane solution with $n$-hexylamine as initiator. ${ }^{5}$ The molar ratio of the anhydride to initiator was 75 . Polymerization occurred at room temperature for $24 \mathrm{~h}$. The PMG obtained was precipitated in dry methanol. A molecular weight of 4400 was estimated from the viscosity measurements in dichloroacetic acid. Poly $(\gamma$-methyl Lglutamate-co-L-glutamic acid)s with $3 \mathrm{~mol} \%$ (MG/GA (97/3)), $8 \mathrm{~mol} \%$ (MG/GA (92/8)), $12 \mathrm{~mol} \%$ (MG/GA (88/12)) $20 \mathrm{~mol} \%$ (MG/GA $(80 / 20)), 26 \mathrm{~mol} \%$ (MG/GA (74/26)) and 30 $\mathrm{mol} \%$ (MG/GA (70/30)) of L-glutamic acid residues were prepared by the saponification of the PMG obtained. ${ }^{6}$ The degree of polymerization did not decrease by saponification. Poly(L-glutamic acid) (PGA, $\left.\bar{M}_{v}=9200\right)$ was kindly provided by Ajinomoto Co., Ltd.

Fluorescent Probes. 8-Anilino-1-naphthalene- sulfonic acid ammonium salt (ANS) was purchased from Wako Chemical Co., Ltd. 1Amino-8-anilinonaphthalene (AN) was synthesized by the reduction of 1-nitro-8-anilinonaphthalene, which was obtained by the nitration of ANS. PMG containing $2.7 \mathrm{~mol}^{\circ} \%$ of $\mathrm{AN}$ in its side chains (AN-PMG) was obtained by the condensation reaction of $\mathrm{MG} /$ GA (97/3) with AN in dimethylformamide, containing dicyclohexylcarbodiimide (DCC) and $n$-hydroxylbenzotriazole (HOBt), for $24 \mathrm{~h}$ at room temperature. ${ }^{7} \mathrm{MG} / \mathrm{GA}$ copolymers and PGA with $3 \mathrm{~mol} \%$ of $\mathrm{AN}$ in their side chains (AN-MG/GAs and AN-PGA) were obtained, respectively, in the same way as AN-PMG. 1-Anilino-8-propionamide-naphthalene (Pro-AN) as a model compound of the AN modified polypeptides was synthesized by the condensation reaction between propionic acid and amino anilino naphthalene using DCC and HOBt. The content of AN in the polypeptides was determined by fluorecence spectroscopic analysis.

Amphiphiles. Distearyldimethylammoniumchloride (DSACl) was kindly provided from Kao Co., Ltd.

Vesicles. The DSACl vesicles containingpolypeptide were prepared as follows. Polypeptides (PMG and $\mathrm{MG} / \mathrm{GAs}$ ) and DSACl were dissolved in chloroform. The residue molar ratio of the polypeptides to $\mathrm{DSACl}$ was fixed at $1.3 \times 10^{-2}$. These solutions were mixed and poured into a glass flusk and then a thin film was formed on the inner surface of the flusk by evaporating the solvent. Tris-HEPES buffer solution ( $\mathrm{pH}$ 6.8) was added to this flusk, and sonicated to $50 \mathrm{~W}$ by Biomic model 7250B ultrasonic processore (SEIKO I \& E/ SONICS \& MATERIALS) for $10 \mathrm{~min}$, under nitrogen atmosphere at $0^{\circ} \mathrm{C}$ to prepare the vesicle. The phase transition temperature of the DSACl vesicle $\left(T_{\mathrm{c}}\right)$ was $40^{\circ} \mathrm{C}$ from differential scanning calorimetry. ${ }^{3}$ 


\section{Methods}

Fluorescence Sepctroscopy. Fluorescence spectra of Pro-AN in various solvents and AN modified polypeptides in the $\mathrm{DSACl}$ vesicle were measured by a spectrofluorophotometer (Shimadzu Co., Ltd., RF-540). The concentration of the DSACl was $0.5 \mathrm{wt} \%$ and that of Pro-AN was $3.0 \times 10^{-5} \mathrm{M}$. The excitation wavelength of $\mathrm{AN}$ was $350 \mathrm{~nm}$.

Permeation Measurements. The DSACl vesicle containing sodium gluconate in the interior was prepared by sonication in the buffer solution containing $0.1 \mathrm{M}$ sodium gluconate in a similar manner as above. The aqueous suspension of the vesicle was filtered off, and $150 \mathrm{mg}$ of the residue were added to the aqueous solution of potassium gluconate $(0.1 \mathrm{M}, 50 \mathrm{ml})$. The resulting vesicle had an ionic concentration gradient between the interior $(0.1 \mathrm{M}$ sodium ion) and exterior $(0.1 \mathrm{M}$ potassium ion), under isotonic conditions. The addition of polypeptides to the vesicle was performed by addition of dimethylformamide solution of polypeptides $\left(1 \mathrm{mg} \mathrm{ml}{ }^{-1}\right)$. The amount of additional polypeptides was $6.8 \times$ $10^{-9}$ mol. The amount of sodium ion that transported across the vesicular bilayer before and after the addition of polypeptides, was detected with an ion meter (Horiba Co., Ltd., N-7ionII).

\section{RESULTS AND DISCUSSION}

\section{Partition of $M G / G A s$ in the DSACl Bilayer Membrane}

We have already reported ${ }^{3}$ that the hydrophobic polypeptide, $\mathrm{PMG}$, is readily incorporated into the bilayer membrane of DSACl. The anionic polypeptide, PGA, could hardly penetrate through the membrane. ${ }^{3}$ Our main concern in this study is the effect of the degree of hydrophobicity of the copolypeptides, MG/ GAs, on their partition between the membrane surface and the membrane interior. It is well known that a fluorescence characteristic of anilinonaphthalene (AN) derivatives is its sensitivity to environmental polarity around the fluorescnece probe. ${ }^{8}$ To elucidate the partition of $\mathrm{MG} / \mathrm{GAs}$ between the polar membrane surface and the hydrophobic interior, the fluorescence spectra of the DSACl bilayer membrane containing $\mathrm{MG} / \mathrm{GAs}$ carrying a small amount of $\mathrm{AN}$ in the side chain were observed. The fluorescence spectra of the DSACl membrane-AN-MG/GA (80/20) system, are shown in Figure 1. It is clear that two emission maxima, $\lambda_{\max }$, appeared at $465 \mathrm{~nm}$ and $490 \mathrm{~nm}$, respectively. Also, in the fluorescence spectra of other systems, except for DSACl-AN-MG/ GA $(70 / 30)$ and AN-PMG, the two $\lambda_{\max }$ could be observed, however, the wavelength of each $\lambda_{\max }$ shifted to the higher one with decreasing hydrophobicity of MG/GA (Figure 2). On the other hand, DSACl-AN-PMG and AN-MG/ GA (70/30) systems each showed only one emission maximum $\lambda_{\max }$ at $453 \mathrm{~nm}$ and $499 \mathrm{~nm}$, respectively (Figure 2). The $\lambda_{\max }$ of AN was shown to be strongly dependent on the polarity around AN. Figure 3 shows the relationship between $\lambda_{\max }$ of Pro-AN, the fluorescent model compound for $\mathrm{AN}$ modified polypeptides, in various solvents and their empirical solvent polarity values, $Z{ }^{9}$ The $\lambda_{\max }$

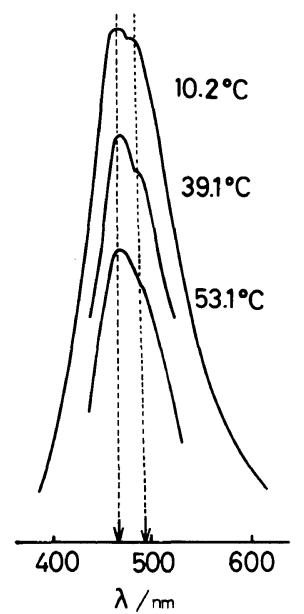

Figure 1. Fluorescence spectra of the DSACl membrane-AN-MG/GA (80/20) system. [AN-MG/GA (80/ $20)] /[\mathrm{DSACl}]=1.3 \times 10^{-2} ;[\mathrm{DSACl}]=0.5 \mathrm{wt} \%$ 


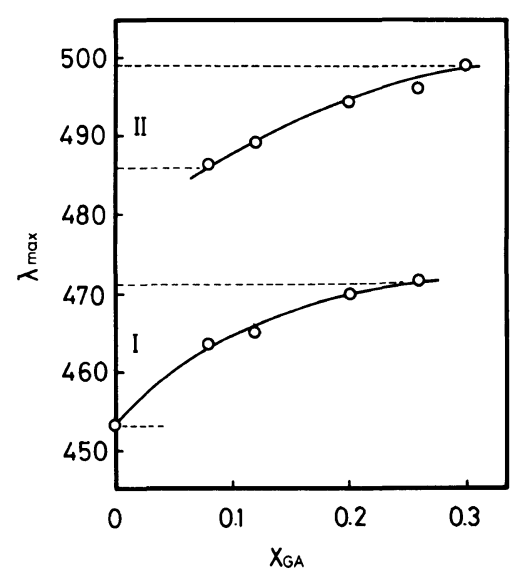

Figure 2. Relation between fluorescent emission maxima of $\mathrm{AN}-\mathrm{MG} / \mathrm{GA}$ and L-glutamic acid content of $\mathrm{MG} / \mathrm{GA}, \quad X_{\mathrm{GA}} . \quad[\mathrm{AN}-\mathrm{MG} / \mathrm{GA}] /[\mathrm{DSACl}]=1.3 \times 10^{-2}$; $[\mathrm{DSACl}]=0.5 \mathrm{wt} \%$.

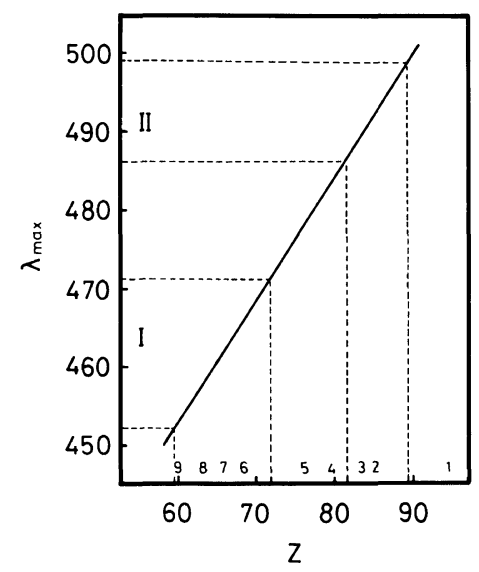

Figure 3. Relation between fluorescent emission maxima of $\mathrm{AN}-\mathrm{MG} / \mathrm{GA}$ and empirical solvent polarity value, $Z$. (1), water; (2), ethylene glycol; (3), methanol; (4), ethanol; (5), iso-propanol; (6), dimethylformamide; (7), acetone; (8), chloroform; (9), octane.

of Pro-AN shifted to a higher wavelength with increasing solvent polarity. ${ }^{10}$ From a comparison of the spectra in Figure 2 with the $\lambda_{\text {max }}$ $-Z$ relation in Figure 3, it was estimated that the values of $\lambda_{\text {max }}$ of $\mathrm{AN}-\mathrm{MG} / \mathrm{GAs}$ at lower and higher wavelength correspond to $Z$ values between octane and dimethylformamide (area I in Figures 2 and 3) and between methanol and water (area II in Figures 2 and 3), respectively. Thus, the emission maxima at lower wavelength (area I in Figure 2) could be assigned to the emission of $\mathrm{AN}-\mathrm{MG} / \mathrm{GAs}$ in the hydrophobic interior of the bilayer and those at higher wavelength (area II in Figure 2) to the emission of the polypeptides at the polar surface of the membrane. Furthermore, it was considered that the red-shifts of both emission maxima were attributable to increase in the polarity of the copolypeptide itself around AN with increasing hydrophilic L-glutamic acid content. The partition equilibrium of $\mathrm{MG} /$ GAs between the DSACl bilayer membrane interior and membrane surface can be represented by eq 1 , with an equilibrium constant $K$, given by eq 2 , respectively.

$$
\begin{aligned}
& \text { (MG/GA) at membrane surface } \\
& \quad \rightleftarrows(\mathrm{MG} / \mathrm{GA}) \text { in membrane interior } \\
& K=(\mathrm{MG} / \mathrm{GA}) \text { in membrane interior } / \\
& \quad(\mathrm{MG} / \mathrm{GA}) \text { at membrane surface }
\end{aligned}
$$

At present, however, we have no methods for accurately estimating the concentration of $\mathrm{MG} / \mathrm{GAs}$ in both regions, membrane surface and interior. The present conditions made it necessary to assume that the concentration of $\mathrm{MG} / \mathrm{GAs}$ in both regions in eq 2 can be roughly estimated from the intensities of lower and higher emission maxima of fluorescence spectra of AN-MG/GAs-DSACl bilayer membrane systems. Here, the emission intensities were corrected by the calibration curve for the emission intensities of Pro-AN in different solvents, considering that the quantum yield of $\mathrm{AN}$ is dependent, at least in part, on polarity around AN. Moreover, $K$ is related to the thermodynamic parameter $\Delta H$ and $\Delta S$ according to

$$
\ln K=-\frac{\Delta H}{R} \cdot \frac{1}{T}+\frac{\Delta S}{R}
$$

where $R$ and $T$ are commonly used notations. Figure 4 shows $\ln K$ for the partition of the MG/GAs between membrane surface and membrane interior as a function of reciprocal of temperatures. The values of $\ln K$ for all plots 


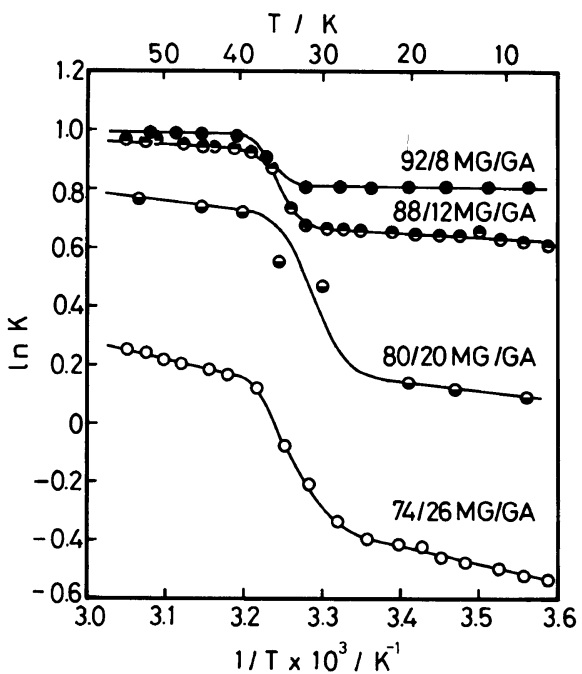

Figure 4. Van't Hoff plots for the partition equilibrium of $\mathrm{MG} / \mathrm{GAs}$ from the $\mathrm{DSACl}$ bilayer membrane surface to the membrane interior.

steeply increased at the gel to the liquid crystalline phase transition temperature, $T_{\mathrm{c}}$, of DSACl bilayer membrane. ${ }^{3}$ The enthalpy $\Delta H$ and entropy $\Delta S$ for the partition below and above $T_{\mathrm{c}}$ can be obtained from the slope and intercept of the linear line in Figure 4 in conjunction with eq 3 . Figure 5 shows the relationship between the value of $\Delta H$ and $\Delta S$ obtained and the glutamic acid content of $\mathrm{MG} / \mathrm{GA}$. The values of $\Delta H$ are positive and almost independent of the phase transition of the DSACl bilayer membrane. This means that the transfer of the anionic copolypeptide containing hydrophobic moieties from the positively charged bilayer membrane surface to the hydrophobic membrane interior is energetically unfavored, i.e., positive energy is required for breaking the electrostatic interaction between the negatively charged L-glutamic acid moiety of MG/GA and positively charged head group of DSACl. On the other hand, the values of $\Delta S$ are strongly dependent on the state of the bilayer membrane, i.e., the values of $\Delta S$ above $T_{\mathrm{c}}$ are considerably larger than that below $T_{\mathrm{c}}$. MG/GA in the membrane interior of liquid crystalline pahse is more

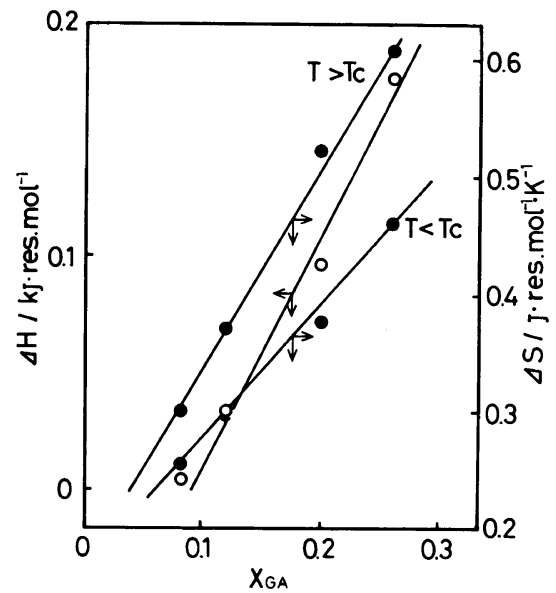

Figure 5. Relationship between the values $\Delta H, \Delta S$ obtained and the L-glutamic acid content of $\mathrm{MG} / \mathrm{GA}$, $X_{\mathrm{GA}}$.

movable owing to its rotational and lateral diffusion, compared with the gel phase of the membrane. This is the main reason why the values of $\Delta S$ for liquid crystalline phase were greater than those of the gel pahse. It is also shown that the values of $\Delta S$ above and below $T_{\mathrm{c}}$ linearly increase with increase in glutamic acid content of $\mathrm{MG} / \mathrm{GA}$. The effect of the $\gamma$-methyl L-glutamate and $L$ glutamic acid moeity in MG/GA on the values of $\Delta H$ and $\Delta S$ can be estimated assuming the following additivity rule.

$$
\begin{gathered}
\Delta H=\Delta H_{\mathrm{MG}}+\left(\Delta H_{\mathrm{GA}}-\Delta H_{\mathrm{MG}}\right) \times X_{\mathrm{GA}} \\
\Delta S=\Delta S_{\mathrm{MG}}+\left(\Delta S_{\mathrm{GA}}-\Delta S_{\mathrm{MG}}\right) \times X_{\mathrm{GA}}
\end{gathered}
$$

where MG and GA denote the $\gamma$-methyl Lglutamate and L-glutamic acid residues, respectively, and $X_{\mathrm{GA}}$ is the $\mathrm{mol} \%$ of glutamic acid of MG/GA. These parameters were obtained from the slope and intercept in Figure 5 in conjunction with eq 4 (Table I). Table I shows the positive value of $\Delta H$ to be attributable to the large positive value of $\Delta H_{\mathrm{GA}}$, reflecting the electrostatic interaction between L-glutamic acid and membrane surface. Therefore, it is concluded that the transfer of $\mathrm{MG} /$ GA from the membrane surface to the membrane interior arises mainly from the entropy 
Table I. Thermodynamic parameters, $\Delta H$ and $\Delta S$ for partition of $\mathrm{MG} / \mathrm{GA}$ between the membrane interior and membrane surface

\begin{tabular}{|c|c|c|c|}
\hline & & MG & GA \\
\hline$\Delta H(\mathrm{KJ} / \mathrm{res} \mathrm{mol})$ & & -0.09 & 0.90 \\
\hline \multirow{2}{*}{$\Delta S\left(\mathrm{KJ} / \mathrm{res} \mathrm{mol} \mathrm{K}^{-1}\right)$} & $\left(T<T_{\mathrm{c}}^{\mathrm{a}}\right)$ & 0.165 & 1.30 \\
\hline & $\left(T>T_{\mathrm{c}}^{\mathrm{a}}\right)$ & 0.168 & 1.85 \\
\hline
\end{tabular}

a $T_{\mathrm{c}}=40^{\circ} \mathrm{C}$.

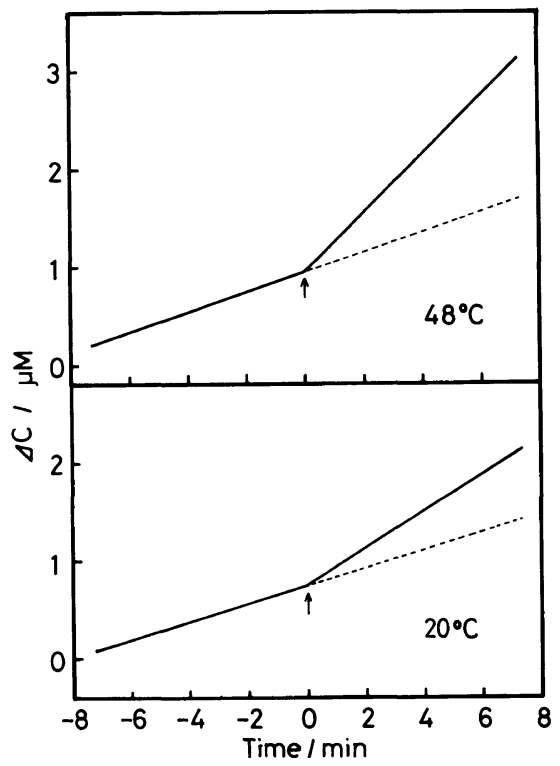

Figure 6. Effect of $\mathrm{MG} / \mathrm{GA}(88 / 12)$ addition on the rate of sodium ion permeation through the $\mathrm{DSACl}$ bilayer membrane at $20^{\circ} \mathrm{C}$ (gel) and $48^{\circ} \mathrm{C}$ (liquid crystal). The arrow marks the time at which the MG/GA $(88 / 12)$ was introduced.

increase occupied by the glutamic acid residue transferred into the liquid crystalline phase.

\section{Ion Permeability through the Bilayer Mem- brane Containing $M G / G A s$}

Figure 6 shows the effect of MG/GA (88/12) addition on the rate of sodium ion permeation through the bilayer membrane from the vesicular interior to the aqueous phase. The ratio of the permeability coefficient, $P / P_{0},\left(P\right.$ and $P_{0}$ are the permeability coefficients of the bilayer

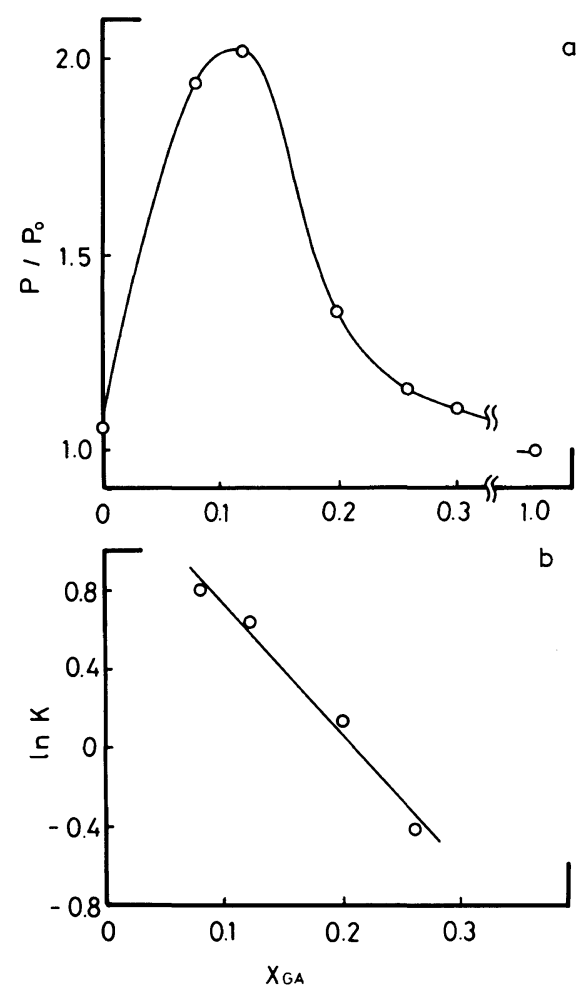

Figure 7. (a): Relation between L-glutamic acid content of $\mathrm{MG} / \mathrm{GA}, X_{\mathrm{GA}}$, and ionic permeability, $P / P_{0}$, at $20^{\circ} \mathrm{C}$. (b): Partition equilibrium constant, $K$, of MG/GA between the membrane interior and membrane surface vs. $X_{\mathrm{GA}}$.

membrane with and without MG/GA (88/12), respectively.) when the membrane is in the gel state $\left(20^{\circ} \mathrm{C}\right)$, could be estimated to be 2.1 . However, that for the liquid crystalline state $\left(48^{\circ} \mathrm{C}\right)$ was 3.0 , i.e., the $P / P_{0}$ for the liquid crystalline state was greater than that for the gel state by a factor of 1.4. Such increase in $P / P_{0}$ can be explained in terms of the increase in the concentration of the MG/GA $(88 / 12)$ in the membrane interior owing to the phase transition from gel to liquid crystalline phase (Figure 4). Thus, the partially charged copolypeptide in the membrane interior, plays an important role in determining the rate of ion permeability, rather than that at the membrane surface. Furthermore, we investigated the effect of the degree of the hydrophilicity of 
MG/GAs on the ion permeability through the bilayer membrane. Figure 7 shows the relationship between the values of $P / P_{0}$ below $T_{\mathrm{c}}$ $\left(20^{\circ} \mathrm{C}\right)$ and L-glutamic acid content. The negatively charged polypeptide, $\mathrm{PGA},{ }^{3}$ and the MG/GA (70/30) with $30 \mathrm{~mol} \%$ of L-glutamic acid residues (Figure 3) are mainly located at the membrane surface below and above $T_{\mathrm{c}}$. It is reasonable, therefore, for the value of $P / P_{0}$ of PGA to be almost unity and that of the $\mathrm{MG} / \mathrm{GA}(70 / 30)$ to be 1.1 . In addition, the value of $P / P_{0}$ was shown to decrease with increase the glutamic acid content of $\mathrm{MG} / \mathrm{GA}$ over $12 \mathrm{~mol} \%$ L-glutamic acid, reflecting the decrease in the concentration of $\mathrm{MG} / \mathrm{GA}$ in the $\mathrm{DSACl}$ membrane interior. In the concentration range less than $12 \mathrm{~mol} \%$, on the other hand, $P / P_{0}$ increased with increase in the $\mathrm{L}-$ glutamic acid content, even though the concentration of $\mathrm{MG} / \mathrm{GAs}$ in the membrane interior decreased. Moreover, it should be noted that the value of $P / P_{0}$ associated with the addition of the hydrophobic polypeptide PMG, which was easily incorporated into the DSACl membrane interior, was almost unity. As a result, $P / P_{0}$ has a maximum value at adequate L-glutamic acid content, $12 \mathrm{~mol} \%$. These results established that sodium ion permeability is determined not only by the concentration of the copolypeptide in the membrane but also by the anionic L-glutamic acid moiety of $\mathrm{MG} / \mathrm{GA}$ in the membrane interior, which may act as a permeation site for the cation.

\section{CONCLUSION}

The partition of the low molecular weight poly(L-glutamic acid) containing various amounts of the hydrophobic $\gamma$-methyl Lglutamate residue, $\mathrm{MG} / \mathrm{GA}$, between the cationic bilayer membrane surface and the hydrophobic membrane interior was investigated by fluorescence spectroscopy. The incorporation of the copolypeptide from the membrane surface into the membrane interior was shown to be energetically unfavored due to electrostatic interactions between $\mathrm{L}$ glutamic acid residues of the copolypeptide and cationic membrane surface of DSACl. The concentration of the copolypeptide, $\mathrm{MG} / \mathrm{GA}$, in the membrane phase thus increased with decreasing L-glutamic acid content of $\mathrm{MG}$ GA. Moreover, the concentration of $\mathrm{MG} / \mathrm{GA}$ in the membrane interior above $T_{\mathrm{c}}$ was higher than that below $T_{\mathrm{c}}$. This shows that, when the membrane is in the liquid crystalline state, the entropy increase of the system resulting from the transfer of $\mathrm{MG} / \mathrm{GA}$ from membrane surface to the membrane interior is much larger than the entropy gain of the system in the gel state. On the other hand, it was also shown that the ion permeability through the DSACl bilayer membrane was strongly dependent on the incorporation of MG/GA into the membrane and effectively increased by the addition of $\mathrm{MG} / \mathrm{GA}$ containing adequate L-glutamic acid content, 12 $\mathrm{mol} \%$ the L-glutamic acid residue. It is concluded, therefore, that the ion permeability through the membrane is dependent not only on the concentration of $\mathrm{MG} / \mathrm{GA}$ in the membrane interior but also on the $\mathrm{L}$ glutamic acid content of MG/GA incorporated into the membrane.

Acknowledgement. We acknowledge the financial support of a Grant-in-Aid from the Ministry of Education, Science, and Culture of Japan.

\section{REFERENCES}

1. P. Hess and R. W. Tsin, Nature, 309, 453 (1972).

2. B. Kriuijiff, A. Rietveld, N. Telder, and B. Baanderager, Biochim. Biophys. Acta, 820, 295 (1985).

3. A. Takizawa, M. Higuchi, T. Kinoshita, and Y. Tsujita, Colloid Polym. Sci., 265, 31 (1987).

4. M. Higuchi, A. Takizawa, T. Kinoshita, and Y. Tsujita, Macromolecules, 20, 2888 (1987).

5. C. H. Bamford, A. Elliot, and W. E. Hanby, "Synthetic Polypeptides," Academic Press, New York, N. Y., 1956. 
6. T. Kinoshita, T. Yamashita, T. Iwata, A. Takizawa, and Y. Tsujita, J. Macromol. Sci. Phys. Ed., B22, 1 (1983).

7. J. L. Houben, A. Fissi, D. Bacciola, N. Rosato, O. Pieroni, and F. Ciardelli, J. Biol. Macromol., 5, 94
(1983).

8. G. K. Radda, Biochem. J., 122, 385 (1971).

9. E. M. Kosower, J. Am. Chem. Soc., 80, 3253 (1958).

10. D. C. Turnner and L. Brand, Biochemistry, 7, 3381 (1967). 